Supporting information

\title{
Biocompatible Two-dimensional Titanium Nanosheets for Multimodal Imaging Guided Cancer Theranostics
}

Zhongjian Xie, Shiyou Chen, Yanhong Duo, Yao Zhu, Taojian Fan, Qingshuang Zou, Mengmeng Qu, Zhitao Lin, Jinlai Zhao, Yang Li, Liping Liu*, Shiyun Bao, Hong Chen*, Dianyuan Fan, and Han Zhang*

Dr. Z. Xie, Dr. S. Chen, Y. Duo, Y Zhu, T. Fan, Q. Zou, Z. Lin, Prof. Fan, Prof. H. Zhang

SZU-NUS Collaborative Innovation Center for Optoelectronic Science \& Technology, International Collaborative Laboratory of 2D Materials for Optoelectronics Science and Technology of Ministry of Education, College of Physics and Optoelectronic Engineering, Shenzhen University, Shenzhen 518060, China

*E-mail: hzhang@szu.edu.cn (H. Zhang).

Q. Zou, Dr. Q. Liu, Dr. Y. Li, Dr. D. Wang, Dr. L. Liu, Dr. S. Bao

Department of Hepatobiliary and Pancreatic Surgery, Shenzhen People's Hospital, Second Clinical Medical College of Jinan University, Shenzhen, Guangdong Province, P. R. China.

*E-mail: leoliping@aliyun.com (L. Liu). 
Dr. Q. Meng

Research Center for Clinical \& Translational Medicine, Beijing 302 Hospital, Beijing, 100039, China

Prof. H. Chen

School of Materials Science and Energy Engineering, Foshan University, Foshan 528000, China

*E-mail: chenhongcs@126.com (H. Chen). 


\section{Experimental Section}

\section{Materials}

Commercially available $\mathrm{Ti}$ powder was purchased from Macklin Company. DSPE-PEG, 5000 Da was bought from Nanocs Inc. (New York, USA). All cell lines were provided by American Type Culture Collection (ATCC). Calcein AM and propidium iodide (PI) assay kits are ordered from Sigma-aldrich. Saline $(0.9 \% \mathrm{NaCl}$ for medical use) was purchased from Sinopharm Chemical Reagent. Other reagents are in analytical grade. Ultrapure water $\left(18.25 \mathrm{M} \Omega . \mathrm{cm}, 25^{\circ} \mathrm{C}\right)$ serve for other water-based dispersions.

\section{Fabrication of TiNSs}

The ultrathin TiNSs were prepared from bulk Ti using liquid-phase exfoliation. Typical exfoliation was mainly divided into two steps: probe sonication and bath sonication. $500 \mathrm{mg}$ of Ti powder was mixed with IPA $(100 \mathrm{ml})$. The suspension was then subjected to probe sonication for $10 \mathrm{~h}$ at the power of $240 \mathrm{~W}$. To avoid over heating during the sonication process, the sonication was set to an on/off cycle of $2 / 4$ seconds and the Ti dispersion was kept in ice water. Subsequently, the Ti dispersion underwent bath sonication at a power of $360 \mathrm{~W}$ for $10 \mathrm{~h}$. The water bath temperature was controlled at $10{ }^{\circ} \mathrm{C}$.

After sonication, the resulting dispersions were centrifuged at $2000 \times \mathrm{g}$ for 30 
min to remove the un-exfoliated component. The supernatant containing the TiNSs was decanted gently and then centrifuged for a further $30 \mathrm{~min}$ at $12000 \times \mathrm{g}$. The precipitate was dried in a vacuum drying oven. To avoid oxidation, the TiNSs were packaged in tinfoil and stored at $4{ }^{\circ} \mathrm{C}$ in the refrigerator for further characterization or use in bio-experiments.

The TiNSs-PEG were further prepared. $1 \mathrm{mg}$ of DSPE-PEG was dispersed in 1 $\mathrm{ml}$ water. $5 \mathrm{ml}$ TiNSs dispersion in water with concentration of $100 \mathrm{ppm}$ was involved sonication for 30 min and then mixed with PEG solution. The mixture underwent bath sonication for several min and stir for $3 \mathrm{~h}$. Then, in order to remove the excess PEG molecules, the resulting mixture was ultrafiltered in Amicon tubes (MWCO 100kDa; Millipore) at $1000 \times \mathrm{g}$ until all water was filtered out, and was washed twice using the same method. The pure TiNSs-PEG were re-suspended in ultrapure water or culture media for further use.

The exfoliation effectiveness of water and IPA was compared through absorbance (Figure S1a). For the same concentration of TiNSs exfoliated by water and IPA, the absorbance presented a large difference. The absorbance of TiNSs exfoliated in IPA was higher than that exfoliated in water. Moreover, the slope of absorbance curve of TiNSs exfoliated in IPA was larger than that exfoliated in water. These two phenomena indicated that the TiNSs exfoliated in IPA has a better dispersion and smaller size than TiNSs exfoliated in water.

The exfoliation process was further characterized by absorbance (Figure S1b). 
As the exfoliation time increased, the absorbance has an overall increase span from UV to NIR region especially from $0 \mathrm{~h}$ to $3 \mathrm{~h}$ and then the exfoliation effectiveness gradually saturated observed from the small increase of absorbance from $3 \mathrm{~h}$ to $8 \mathrm{~h}$.
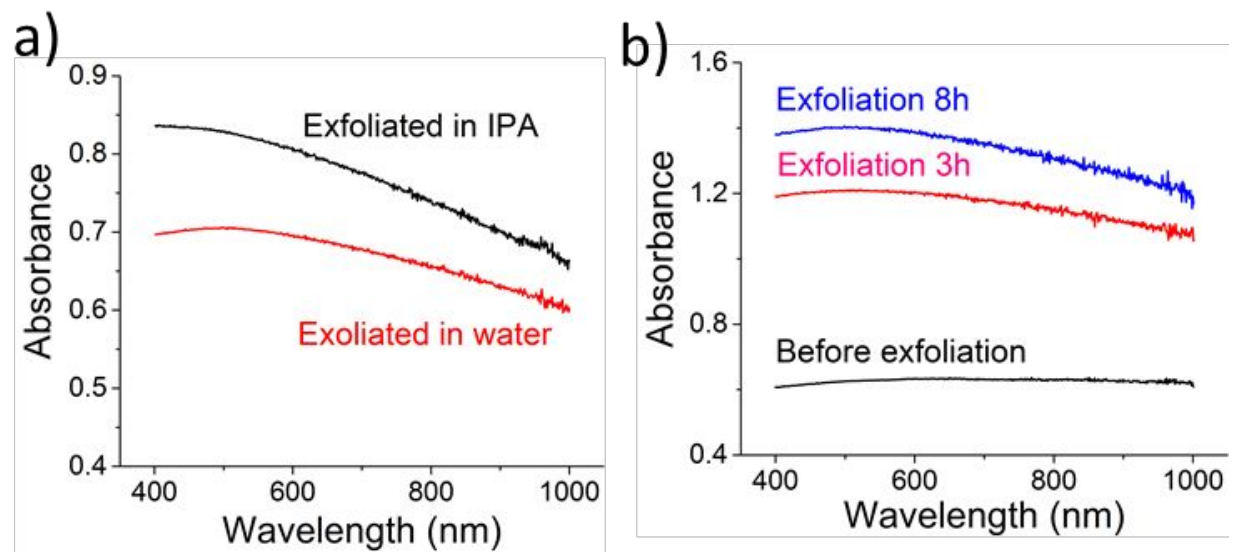

Figure S1. Liquid-phase exfoliation (LPE) for TiNSs. a) For TiNSs exfoliated in water and IPA, different absorbance for the same concentration. b) Exfoliation effectiveness for different exfoliation time shown by the absorbance.

\section{Characterization}

To confirm the three-dimensional morphology of TiNSs, both atomic force microscopy (AFM, Bruker, Dimension Fastscan) and transmission electron microscopy (TEM, JEM1230) were used. AFM samples were prepared by dispersing on a silicon substrate using the spin-coating method and AFM images were scanned at resolution of 512 pixels at one line. HRTEM images and selected-area electron diffraction (SAED) were obtained using a Tecnai G2 F30 with an acceleration voltage of $300 \mathrm{kV}$. X-ray diffraction was characterized using a Philips X'Pert Pro Super 
diffractometer. X-ray photoelectron spectra were characterized using a VG Escalab MK II spectrometer. Broadband absorbance was measured in the range of 400-1000 nm using a Cary 60 spectrometer from Agilent. The Fourier transform infrared (FTIR) spectrum was measured to verify the PEG coating of TiNSs. An $808 \mathrm{~nm}$ fiber-coupled continuous semiconductor diode laser, LSR808H from Lasever Inc., was used as the laser source for the photothermal experiments. For the photothermal temperature measurements, an infrared thermal imaging camera (FLIR E-60) and thermocouple were used. The content of Ti element was quantitatively measured by inductively coupled plasma-mass spectrometry (ICP-MS, Agilent 7700).

Different sized TiNSs can be obtained through different centrifugation force. Two kinds of TiNSs were obtained through choosing centrifugation force of $1000 \mathrm{~g}$ and 2000 g, namely large TiNSs and small TiNSs. The large TiNSs and the small TiNSs were compared in Figure S2. As shown by the TEM image (Figure S2a), the large TiNSs are in the lateral size of 100-200 nm, while the size of small TiNSs is less than $50 \mathrm{~nm}$ (Figure S2b). Moreover, the color of large TiNSs is obviously darker than small TiNSs, indicating the thicker and large TiNSs. In Figure S2c and d, different concentrations of TiNSs dispersed in water were shown. For the same concentration, it was obviously observed that the dispersion of small TiNSs was darker than that of large TiNSs, indicating the stronger absorption and better dispersibility of small TiNSs than large TiNSs. 

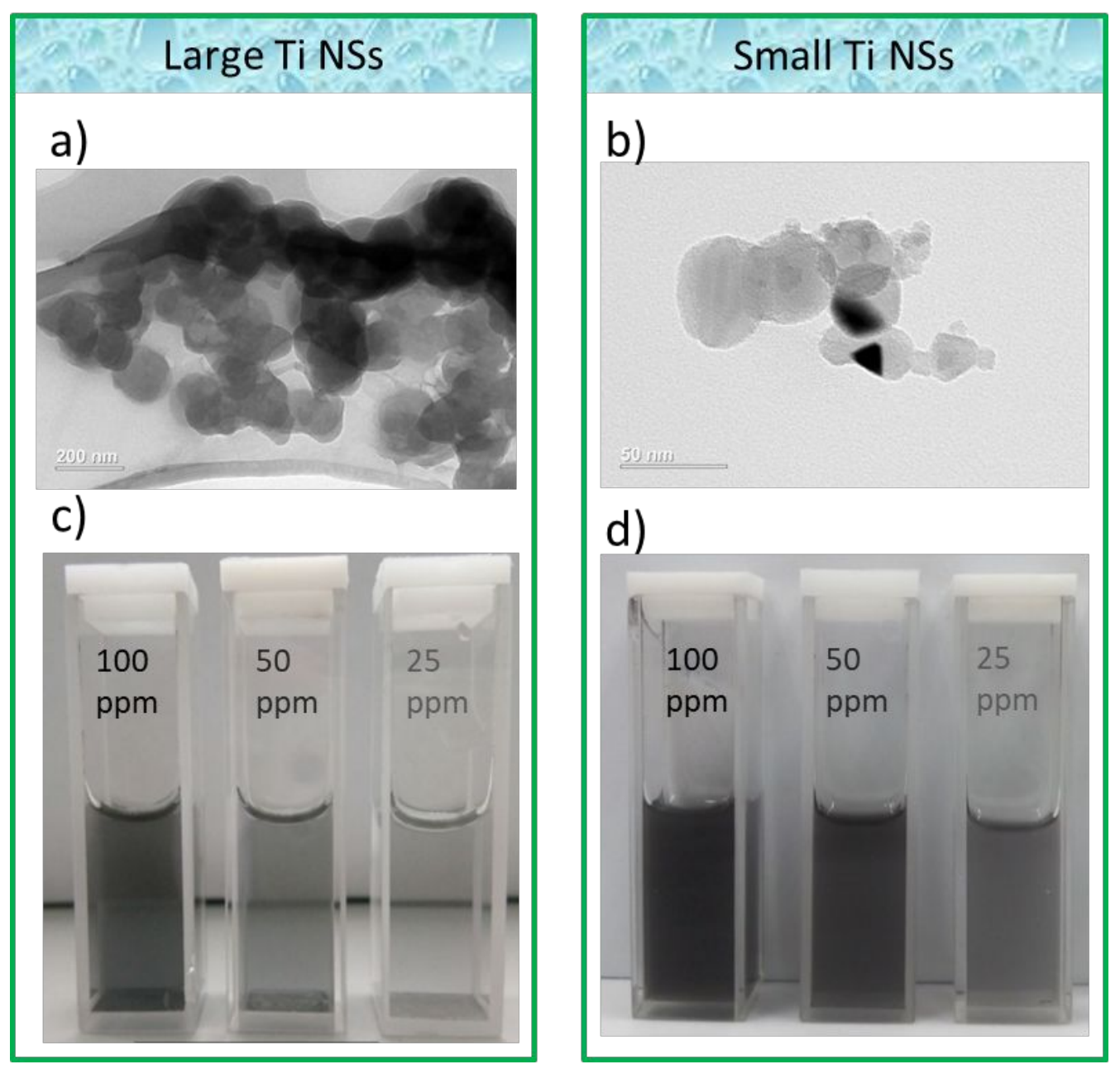

Figure S2. Comparison of large TiNSs and small TiNSs. TEM image for a) large TiNSs and b)

small TiNSs. Comparison of the same concentration for c) large TiNSs and d) small TiNSs dispersed in water.

The absorbance for the same concentration of large and small TiNSs was compared (Figure S3a). It was found that the absorbance of small TiNSs was much higher than that of large TiNSs for the same concentration. Moreover, the slope of absorbance curve for small TiNSs was larger than that of large TiNSs, indicating the different absorbance of different sized TiNSs as observed from Figure S2c and d. Corresponding to the absorbance, the extinction coefficient and photothermal temperature change of large TiNSs were also lower than the small TiNSs (Figure S3c 
and d). Consequently, the small TiNSs with lateral size of less than $\sim 50 \mathrm{~nm}$ has a better photothermal performance and used for bio-experiments.

a)

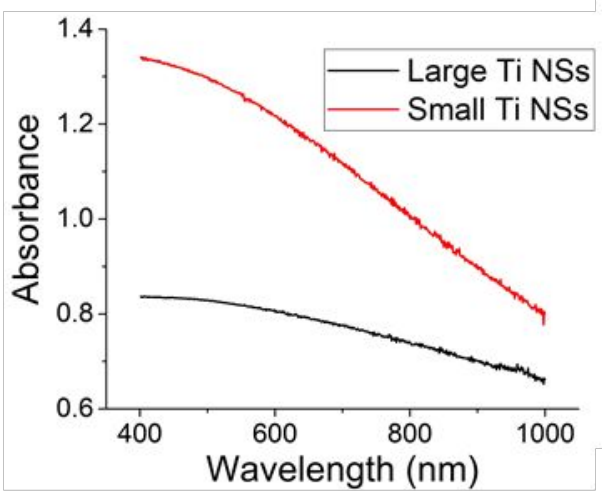

c)

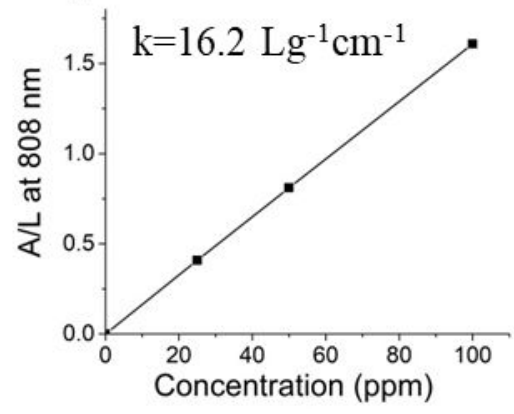

b)

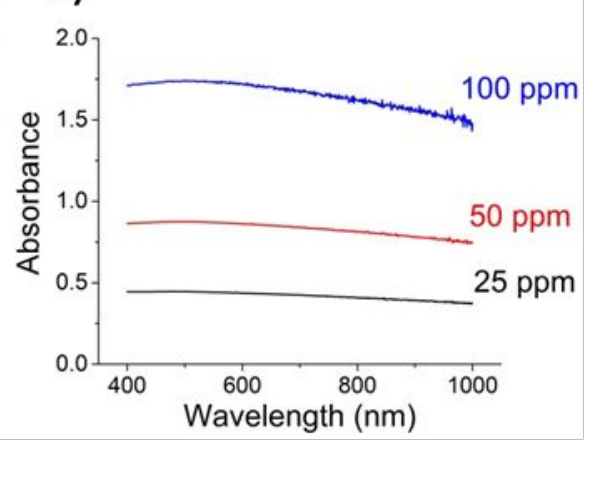

d)

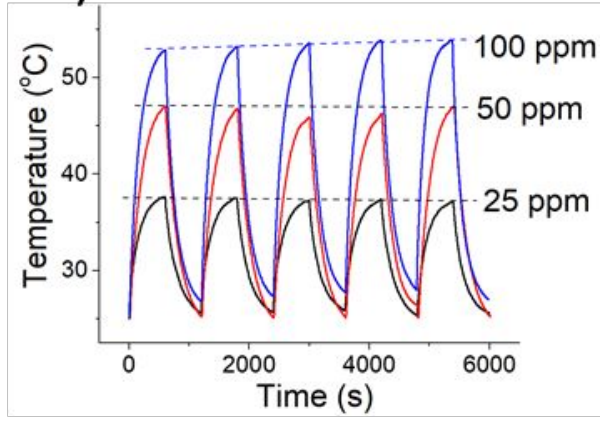

Figure S3. Photothermal performance of large TiNSs. a) Different absorbance of large and small TiNSs for the same concentration. b) Absorbance at different concentrations of large TiNSs. c) Extinction coefficient of large TiNSs. d) Photothermal temperature change for different concentrations.

\section{Photothermal conversion efficiency (PTCE)}

The photothermal experiment was conducted on a custom setup. $1 \mathrm{~mL}$ of the TiNSs dispersion in water was contained in a standard quartz cuvette with $1 \mathrm{~cm}$ path length.

A fiber-coupled continuous semiconductor diode laser $(808 \mathrm{~nm}$, LSR808H, Lasever Inc. Jiangsu, China) was used to provide the laser source and an infrared thermal 
imaging camera (FLIR E-60) was used to monitor the photothermal temperature change.

\section{Biodegradability of TiNSs.}

The degradability of TiNSs-PEG in PBS was measured. It was found that the dispersion became almost transparent, suggesting the degradability of TiNSs-PEG (Figure S4a). In the meantime, the TEM image can also demonstrate the degradability (Figure S4b).

a)

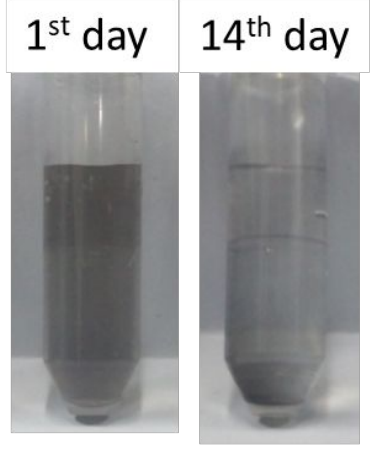

b)

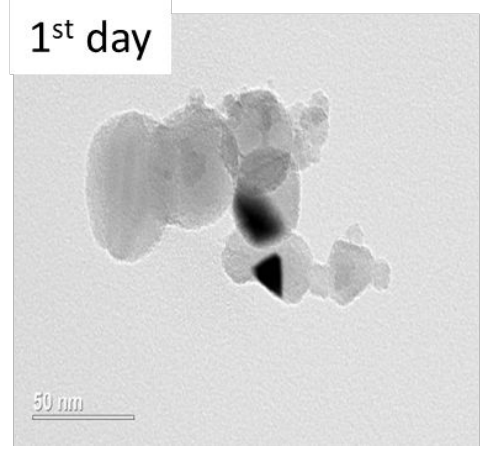

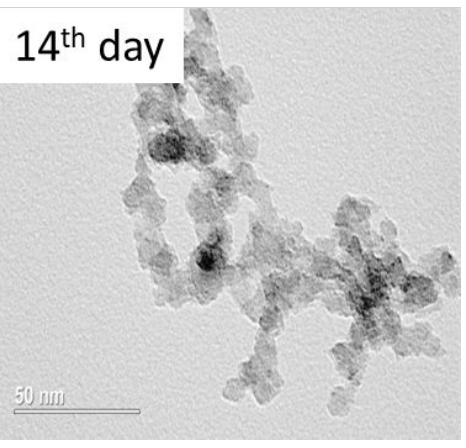

Figure S4. Degradability of TiNSs-PEG in PBS shown by the a) photograph and b) TEM images.

Moreover, the dispersion stability of TiNSs and TiNSs-PEG in PBS were tested and it was found that the TiNSs obtained a super stability without sediment after 2 days but TiNSs almost precipitated, as shown in Figure S5.

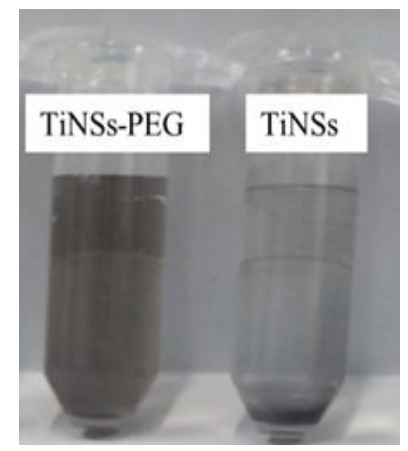

Figure S5. Comparison of the stability of TiNSs and TiNSs-PEG in PBS. 


\section{Cell Culture}

Mouse melanoma cells (B16) and mouse macrophage cells (J774A.1) were incubated in Dulbecco's Modified Eagle Medium (DMEM) with high concentrated glucose (Hyclone). Human hepatocellular carcinoma cells (SMMC-7721) were incubated in a mixture of Ham's F-12 medium (Hyclone) and DMEM with mixture ratio of 1:1. 1\% Pen/Strep (Gibco) and 10\% fetal bovine serum (Gibco) were used to supplement the culture medium and the culture condition is $5 \% \mathrm{CO}_{2}$ at $37^{\circ} \mathrm{C}$.

\section{In vitro Experiments}

Cells seeded in 96-well plates were treated with TiNSs, TiNSs-PEG, or left untreated (Mock). For in vitro cytotoxicity assays, cells were directly subjected to cell-counting kit (CCK8) tests (Beyotime Biotechnology) 24 hours post TiNSs incubation. For in vitro photothermal study, cells were coexisted with TiNSs for $4 \mathrm{~h}$ and were then experienced a radiation using $808 \mathrm{~nm}$ laser with power density of $1.0 \mathrm{~W} \mathrm{~cm}-2$ for 5 min. Finally, the cells went through the CCK8 tests following the manufacturer instructions $24 \mathrm{~h}$ post irradiation. The relative cell viability was normalized to the Mock samples (concentration of TiNSs $=0 \mathrm{ppm}$ ) of each cell line. To show the photothermal killing effect, cells were subjected to calcein AM/PI staining (Sigma) 6 hours post irradiation. The live cells and dead cells appear to be green and red color stained by calcein AM and PI, respectively.

The targeting experiments in vitro towards cancer cells (SMMC-7721) and 
normal cells (HL-7702) were conducted. Confocal laser scanning microscopy (CLSM) images demonstrated the efficient intracellular uptake of Cy5.5-labeled PEG-TiNSs by the SMMC-7721, while a low uptake degree by the HL-7702 (Figure S6).

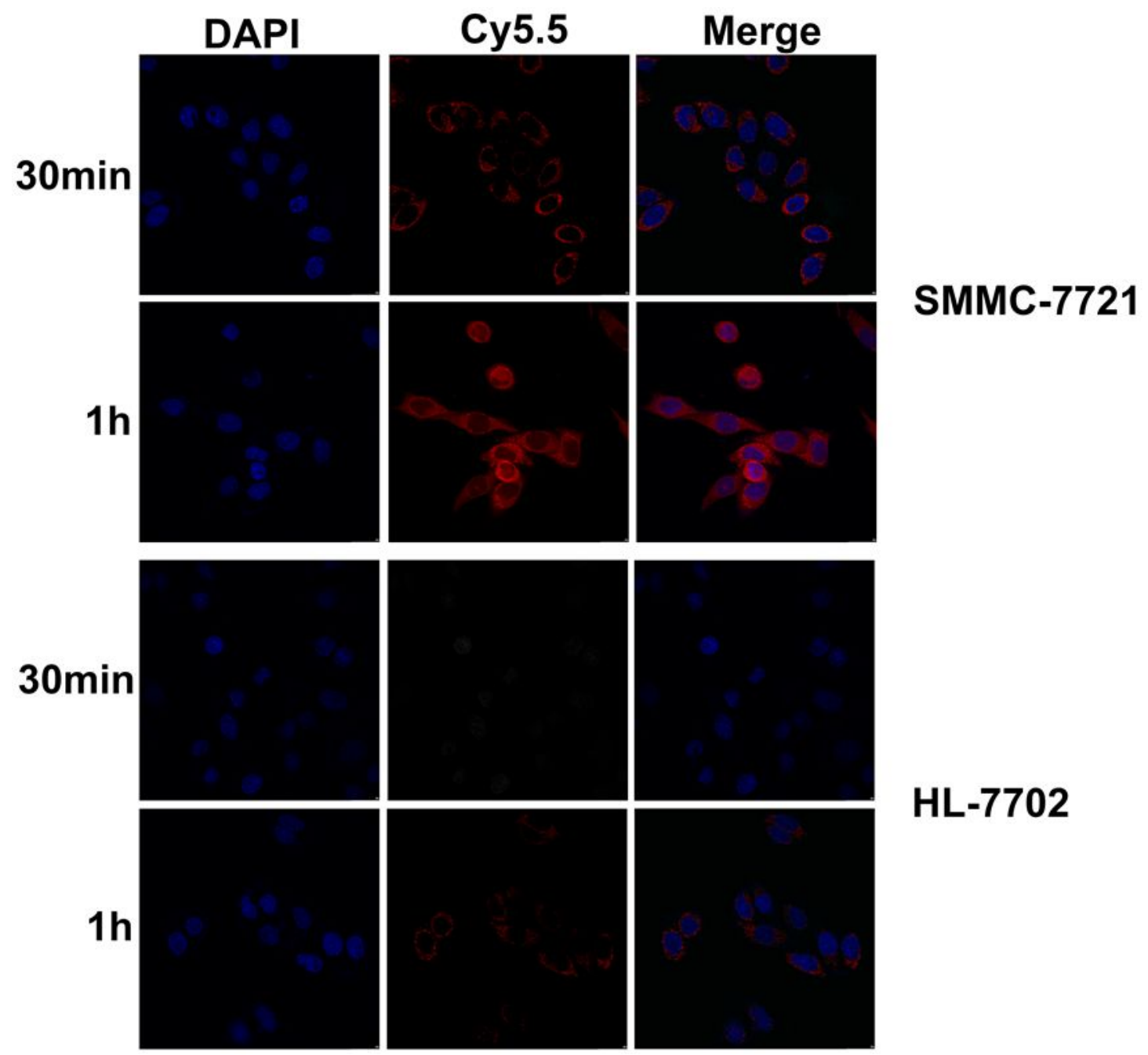

Figure S6. Cell targeting experiments.

\section{Mouse Experiments}

All of the animal experiments were allowed by the Animal Welfare and Research Ethics Committee at Shenzhen University (ID: 2017003). The mice were ordered 
from Guangdong Medical Laboratory Animal Center (Guangzhou, China). 6-week old female Balb/c nude mice were used for toxicity assays and establishment of tumor-bearing mouse models. For PTT experiments, human hepatocellular carcinoma mouse models were built by subcutaneously injecting $2 * 10^{6}$ SMMC-7721 cells for each mouse. For PA and CT imaging, human cervical tumor mouse models were established by injection of $2 * 10^{6} \mathrm{HeLa}$ cells in subcutaneous tissue for each mouse. Mouse anaesthesia was achieved by intraperitoneal injection of sodium pentobarbital $(50 \mathrm{mg} / \mathrm{kg}$ ) solution (dissolved in $0.9 \% \mathrm{NaCl}$ solution). Mice were euthanized before the ACUSU maximum allowable tumor burden of $2 \mathrm{~cm}^{3}$.

\section{In vivo Toxicity}

For all in vivo studies, the TiNSs-PEG were dispersed in saline for in vivo injection. Mice were randomly distributed into 3 groups ( $\mathrm{n}=5$ each group) and were intravenously injected with saline, TiNSs and TiNSs-PEG at first day, respectively. The dose of TiNSs or TiNSs-PEG was $5 \mathrm{mg} \mathrm{kg}^{-1}$. To monitor the in vivo toxicity, body weight was measured every 2 days until the $19^{\text {th }}$ day. The mice were then euthanized and the major organs of heart, liver, spleen, lung and kidney were incised for H\&E staining. ${ }^{1}$

Furthermore, the degradation of TiNSs in vivo was proved at different time points after intravenous injection (Figure S7). The quantity of TiNSs in main organs firstly increases from $1 \mathrm{~h}$ to $12 \mathrm{~h}$ and then decreases from $12 \mathrm{~h}$ to 7 day, which is mainly 
shown in liver and spleen, indicating the bio-degradation of TiNSs in vivo.

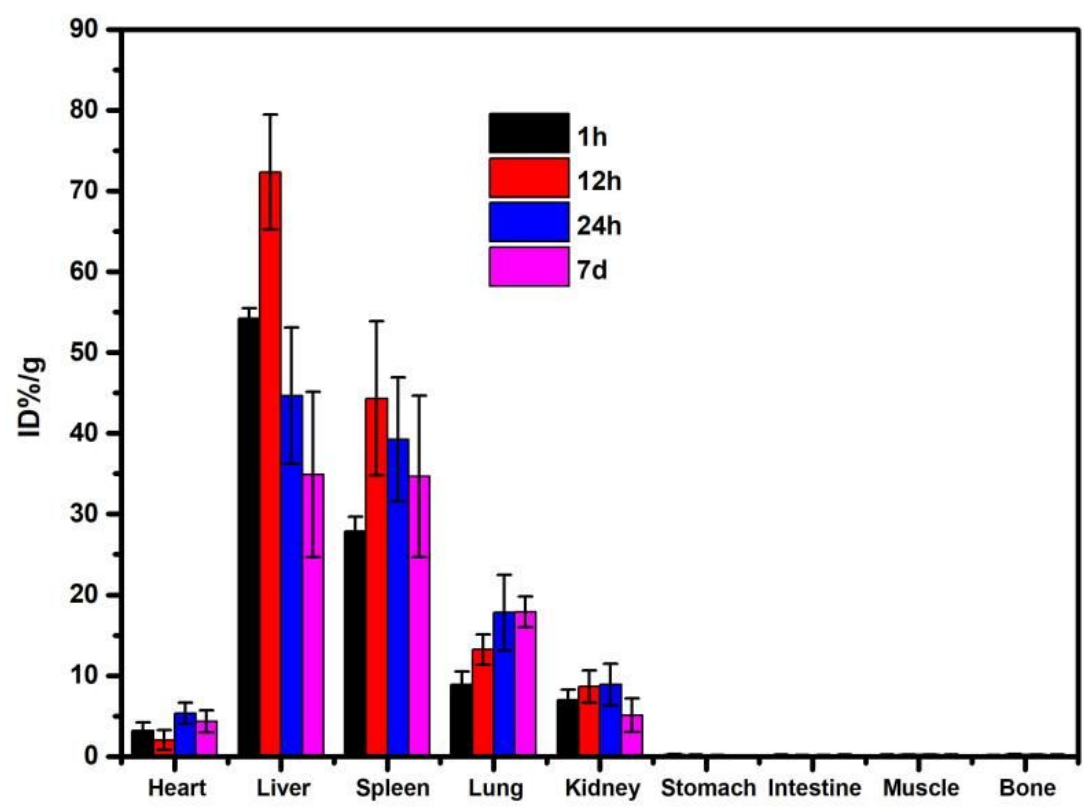

Figure S7. Biodegradation of TiNSs in main organs in different time intervals.

\section{Dual-Modal in vivo PA and CT imaging}

For in vivo PA imaging, the cervical-tumor-holding mice were intravenously treated with $200 \mu \mathrm{L}$ of TiNSs-PEG samples $\left(5 \mathrm{mg} \mathrm{mL}^{-1}\right)$. After the treatment, the PA experiments were conducted at different time $(0,1,2,4,8,24$, and $48 \mathrm{~h})$. For in vivo CT imaging, $200 \mu \mathrm{L}$ of TiNSs-PEG $\left(5 \mathrm{mg} \mathrm{mL}^{-1}\right)$ was intravenously injected. The mice were set on CT equipment to image before injection and post injection for $24 \mathrm{~h}$.

The PA imaging data were recorded by Nexus 128 photoacoustic equipment (Endra Life Sciences). The equipment is equipped with a tunable ns pulsed laser (pulse frequency of $20 \mathrm{~Hz}, 7 \mathrm{~ns}$ pulses, $7 \mathrm{~mJ}$ per pulse at the mouse skin and wavelength from 680 to $950 \mathrm{~nm}$ ). The CT imaging was operated on GE discovery CT750 HD (GE Healthcare). 
The PA images for dispersions of TiNSs show the gradually enhanced PA signals as concentrations increases (Figure S8a, b). The CT values show also an enhanced contrast performance with the increasing concentration. The CT ability of TiNSs can be shown by the slope value of 105. $26 \mathrm{HU} \mathrm{L} \mathrm{g}^{-1}$ (Figure S8c, d).

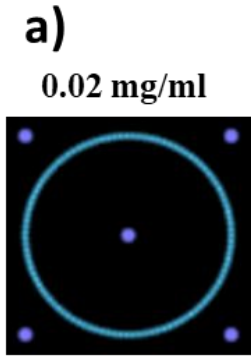

$1 \mathrm{mg} / \mathrm{ml}$

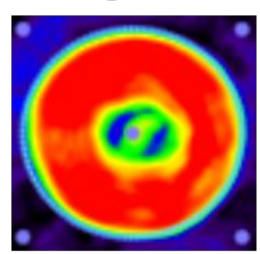

c)

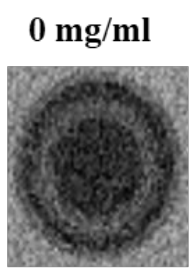

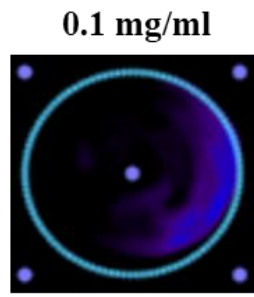

$3 \mathrm{mg} / \mathrm{ml}$

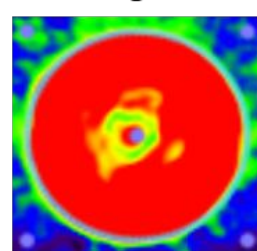

$0.2 \mathrm{mg} / \mathrm{ml}$

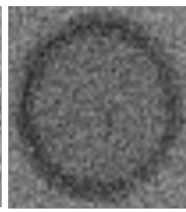

$0.4 \mathrm{mg} / \mathrm{ml}$

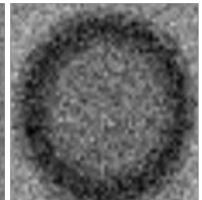

$0.5 \mathrm{mg} / \mathrm{ml}$

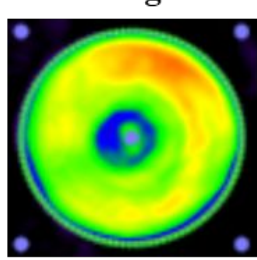

$6 \mathrm{mg} / \mathrm{ml}$

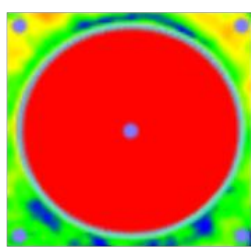

$0.8 \mathrm{mg} / \mathrm{ml}$

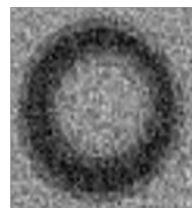

b)

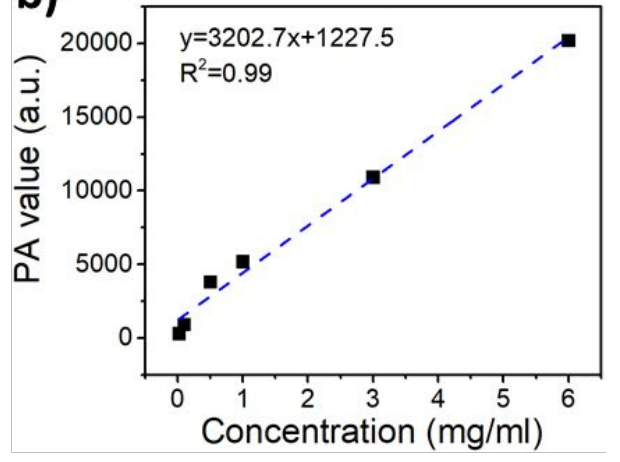

d)

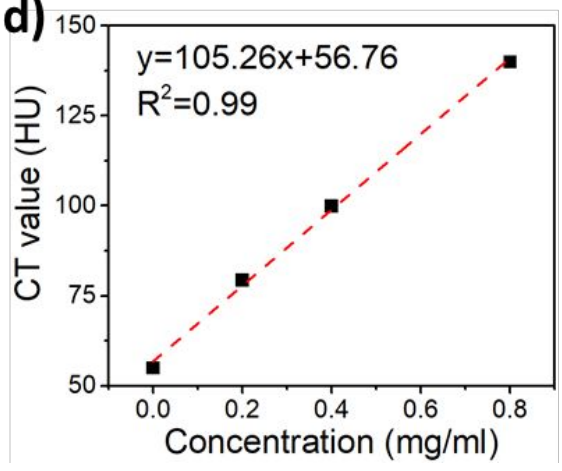

Figure S8. a) PA images of TiNSs. b) PA values. c) CT images of TiNSs. d) CT values.

\section{In vivo Photothermal Tumor Therapy}

The mice were randomly distributed into 4 groups ( $\mathrm{n}=5$ for each group) for different treatments by intravenous injection: group 1, saline; group 2, TiNSs-PEG; group3, saline with NIR irradiation; group 4, TiNSs-PEG with NIR irradiation. The TiNSs-PEG was injected intravenously in dosage of $2 \mathrm{mg} \mathrm{kg}^{-1} .24 \mathrm{~h}$ post injection, the mice were anaesthetized and the tumors were irradiated by the $808 \mathrm{~nm}$ laser $(1.0 \mathrm{~W}$ 
$\left.\mathrm{cm}^{-2}, 5 \mathrm{~min}\right)$. The temperature of the tumor was measured using a thermography. After the photothermal treatment, the tumor volumes and body weights were recorded every 2 days. Tumor size was measured using a caliper and the volumes were calculated through the equation (volume $=$ length $\times$ width $\left.2 / 2, V=1 \times \mathrm{w}^{2} / 2\right)$. At $19^{\text {th }}$ day, all of the mice were euthanized and the organs of heart, liver, spleen, lung and kidney were isolated for H\&E staining to assess the possible damage caused by the PTT.

\section{Statistical Analysis}

All of the data were handled using Graphpad Prism software and are presented as means \pm SD. For in vitro studies, cell viability was normalized to the mean of Mock samples for each cell line, which was set to be $100 \%, n=3$ biological replicates. Analysis of significance was performed by student's $t$-tests. For animal experiments, the significance analysis of tumor volumes between the four groups was performed using multiple $t$-tests, $\mathrm{n}=5$ for each group. $p<0.05$ was considered statistically significant, n.s. means non-significant, ${ }^{*} p<0.05,{ }^{*} p<0.01, * * * p<0.001$.

\section{Haematology tests}

The normal haematology tests were conducted by measuring red blood cells (RBC), white blood cells (WBC), platelets (PLT), haemoglobin (HGB), mean corpuscular haemoglobin $(\mathrm{MCH})$, mean corpuscular haemoglobin concentration (MCHC), haematocrit (HCT) and mean corpuscular volume (MCV) (Figure S9). It was observed that there were no evident changes from TiNSs-treated groups in contrast 
with the control group, indicating no inflammation and other negative impact on blood and organs induced by TiNSs.

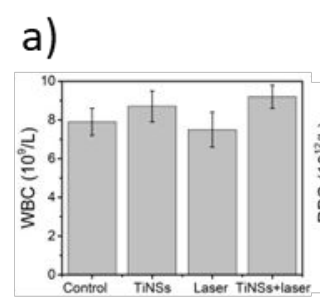

\section{b)}

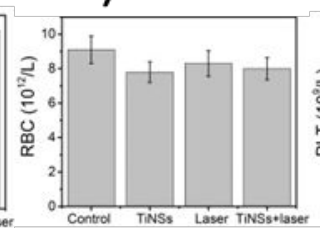

e)
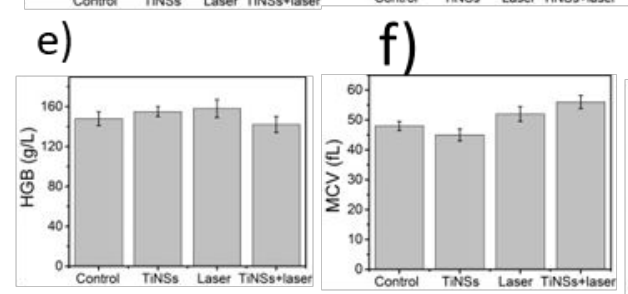

c)

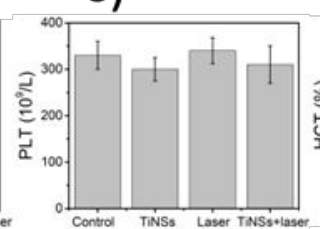

d)

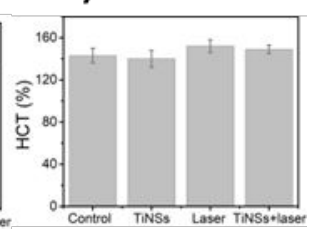

h)

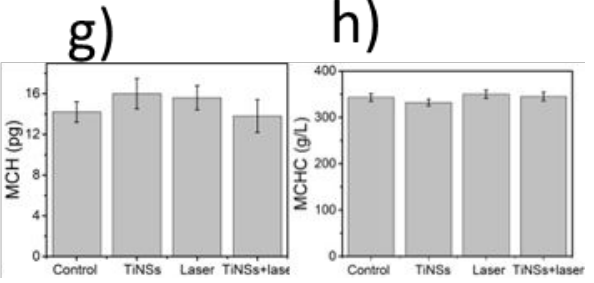

Figure S9. Hematological parameters of the mice after intravenous administration of TiNSs, including a) WBC, b) RBC, c) PLT, d) HCT, e) HGB, f) MCV, g) MCH, and h) MCHC.

\section{References}

(1) Tao, W.; Zhu, X.; Yu, X.; Zeng, X.; Xiao, Q.; Zhang, X.; Ji, X.; Wang, X.; Shi, J.;

Zhang, H. Black Phosphorus Nanosheets as a Robust Delivery Platform for Cancer

Theranostics. Adv. Mater. 2017, 29, 1603276. 